\title{
An Explicit Single-Step Nonlinear Numerical Method for First Order Initial Value Problems (IVPs)
}

\author{
Omolara Fatimah Bakre1, Ashiribo Senapon Wusu², Moses Adebowale Akanbi ${ }^{2}$ \\ ${ }^{1}$ Department of Mathematics \& Statistics, Federal College of Education (Technical), Lagos, Nigeria \\ ${ }^{2}$ Department of Mathematics, Lagos State University, Lagos, Nigeria \\ Email: larabakre@gmail.com,wussy_ash@yahoo.com, moses.akanbi@lasu.edu.ng
}

How to cite this paper: Bakre, O.F., Wusu, A.S. and Akanbi, M.A. (2020) An Explicit Single-Step Nonlinear Numerical Method for First Order Initial Value Problems (IVPs). Journal of Applied Mathematics and Physics, 8, 1729-1735.

https://doi.org/10.4236/jamp.2020.89130

Received: July 24, 2020

Accepted: August 31, 2020

Published: September 3, 2020

Copyright $\odot 2020$ by author(s) and Scientific Research Publishing Inc. This work is licensed under the Creative Commons Attribution International License (CC BY 4.0).

http://creativecommons.org/licenses/by/4.0/

\begin{abstract}
Interest in the construction of efficient methods for solving initial value problems that have some peculiar properties with it or its solution is recently gaining wide popularity. Based on the assumption that the solution is representable by nonlinear trigonometric expressions, this work presents an explicit single-step nonlinear method for solving first order initial value problems whose solution possesses singularity. The stability and convergence properties of the constructed scheme are also presented. Implementation of the new method on some standard test problems compared with those discussed in the literature proved its accuracy and efficiency.
\end{abstract}

\section{Keywords}

Ordinary Differential Equations, First Order, Initial Value Problems, Nonlinear, Singularities

\section{Introduction}

Many of the numerical methods for obtaining the solution of the first order ordinary differential equation

$$
y^{\prime}=f(x, y(x)), x \in\left[x_{0}, X\right], y\left(x_{0}\right)=\eta
$$

are based on the assumption that the solution is locally representable by a polynomial. However, when a given initial value problem or its theoretical solution $u(t)$ is known to posse a singularity, then it is particularly inappropriate to represent $y(x)$, in the neighbourhood of the singularity by a polynomial [1] [2]. This is evident as Runge-Kutta type methods, Obrechkoff methods and general linear multistep methods usually produce very poor solutions around singularity points [1] [3] [4] [5]. The authors in [4] were the first to develop quadrature formulas based on rational interpolating functions. On the other hand, the 
rational interpolation schemes proposed in [2], was seen to be effective in the neighbourhood of the singularity and even beyond as reiterated in [5]. The work of authors in [4] was modified by [2] with a replacement of the general rational function Luke et al. replaced the general rational function of [4] by $F(x)=\frac{P_{m}(x)}{Q_{n}(x)}$ where $P_{m}(x)$ and $Q_{n}(x)$ are respectively polynomial of degree $m$ and $n$. The resulting schemes require analytic generation of first and higher order derivatives, hence, the major limitation of the schemes. Since rational functions are more appropriate for the representation of functions close to singularities than polynomials, the limitation is overcome by a local representation of the theoretical solution with a rational expression. Interestingly, this approach appears to be promising as several methods are now being constructed in this direction [6] [7] [8] [9] [10]. The works of the authors in [6] [7] [9] [10] [11] [12] showed that solution around singularity point are well approximated by this approach. In this work, an explicit single-step nonlinear method involving higher derivatives of the state function for solving (1) is presented. The local truncation error and absolute stability of the new method are also discussed.

\section{Construction of Method}

In this work, we assumed that the theoretical solution $y(x)$ of (1) can locally be represented by a rational interpolant $r(x)$, of the form

$$
r(x)=\frac{a_{0}+a_{1} x+a_{2} x^{2}+a_{3} x^{3}+a_{4} x^{4}}{b_{0}+x} .
$$

To construct an explicit single-step method with (2) for (1), it requires that $r(x)$ satisfies the following:

$$
\left.\begin{array}{l}
r\left(x_{n+j}\right)=y_{n+j}, \quad j=0,1, \\
r^{(i)}\left(x_{n+j}\right)=y_{n+j}^{(i)}, \quad j=0, \quad i=0,1,2,3,4,5 .
\end{array}\right\}
$$

Substituting for expressions and simplifying (3) yields

$$
\left.\begin{array}{l}
y_{n}=\frac{a_{4} x_{n}^{4}+a_{3} x_{n}^{3}+a_{2} x_{n}^{2}+a_{1} x_{n}+a_{0}}{b_{0}+x_{n}} \\
y_{n+1}=\frac{a_{4}\left(h+x_{n}\right)^{4}+a_{3}\left(h+x_{n}\right)^{3}+a_{2}\left(h+x_{n}\right)^{2}+a_{1}\left(h+x_{n}\right)+a_{0}}{b_{0}+h+x_{n}} \\
y_{n}^{\prime}=\frac{4 a_{4} b_{0} x_{n}^{3}+3 a_{3} b_{0} x_{n}^{2}+2 a_{2} b_{0} x_{n}+a_{1} b_{0}+3 a_{4} x_{n}^{4}+2 a_{3} x_{n}^{3}+a_{2} x_{n}^{2}-a_{0}}{\left(b_{0}+x_{n}\right)^{2}} \\
y_{n}^{(2)}=2\left(\frac{b_{0}\left(b_{0}\left(a_{4} b_{0}^{2}-a_{3} b_{0}+a_{2}\right)-a_{1}\right)+a_{0}}{\left(b_{0}+x_{n}\right)^{3}}-a_{4} b_{0}+3 a_{4} x_{n}+a_{3}\right) \\
y_{n}^{(3)}=6 a_{4}-\frac{6\left(b_{0}\left(b_{0}\left(a_{4} b_{0}^{2}-a_{3} b_{0}+a_{2}\right)-a_{1}\right)+a_{0}\right)}{\left(b_{0}+x_{n}\right)^{4}} \\
y_{n}^{(4)}=\frac{24\left(a_{4} b_{0}^{4}-a_{3} b_{0}^{3}+a_{2} b_{0}^{2}-a_{1} b_{0}+a_{0}\right)}{\left(b_{0}+x_{n}\right)^{5}} \\
y_{n}^{(5)}=\frac{120\left(a_{4} b_{0}^{4}-a_{3} b_{0}^{3}+a_{2} b_{0}^{2}-a_{1} b_{0}+a_{0}\right)}{\left(b_{0}+x_{n}\right)^{6}}
\end{array}\right\}
$$


Eliminating the undetermined coefficients $a_{0}, a_{1}, a_{2}, a_{3}, a_{4}$ and $b_{0}$ in (4) results in

$$
y_{n+1}=y_{n}+h y_{n}^{\prime}+\frac{1}{2} h^{2} y_{n}^{\prime \prime}+\frac{1}{6} h^{3} y_{n}^{(3)}-\frac{5 h^{4}\left(y_{n}^{(4)}\right)^{2}}{24\left(h y_{n}^{(5)}-5 y_{n}^{(4)}\right)} .
$$

The resulting method (5) is explicit, self-starting and nonlinear. We shall refer to (5) as $N L M 4$ which is the method proposed in this work. The new method NLM4 is suitable for solving initial value problems whose solution possesses singularities.

\section{Local Truncation Error and Absolute Stability of Constructed Method}

In this section, the local truncation error (lte) and the absolute stability properties of the new method proposed in this work are considered.

\subsection{Local Truncation Error}

Local Truncation Error. The local truncation error $T_{n+1}$ at $x_{n+1}$ of the general explicit one step method

$$
y_{n+1}=y_{n}-h \phi\left(x_{n}, y_{n}, h\right)
$$

is given as

$$
T_{n+1}=y\left(x_{n+1}\right)-y\left(x_{n}\right)-h \phi\left(x_{n}, y\left(x_{n}\right), h\right)
$$

where, $y\left(x_{n}\right)$ is the theoretical solution. Using the above definition, it follows that the local truncation error of the constructed one step method can be written as

$$
T_{n+1}=y\left(x_{n+1}\right)-y_{n+1}
$$

\subsection{Order of a Ordinary Differential Equation}

Order of a Ordinary Differential Equation: A numerical method is said to be of order $p$ if $p$ is the largest integer for which $T_{n+1}=\mathcal{O}\left(h^{p+1}\right)$ for every $n$ and $p \geq 1$. Following the above definition, the local truncation error of the method constructed in this work is obtained as the residual when $y_{n+1}$ is replaced by $y\left(x_{n+1}\right)$. Below is the local truncation error for the method constructed in this work.

$$
T_{n+1}=\frac{1}{600 y^{(4)}}\left(h^{6}\left(y^{(5)}\right)^{2}\right)
$$

\subsection{Consistency}

A scheme is said to be consistent if the difference equation of the integrating formula exactly approximates the differential equation it intends to solve as the step size approaches zero. In order to establish the consistency property of the constructed method, it is sufficient to show that 


$$
\lim _{h \rightarrow 0} \frac{y_{n+1}-y_{n}}{h}=0
$$

Now,

$$
\lim _{h \rightarrow 0} \frac{y_{n+1}-y_{n}}{h}=\lim _{h \rightarrow 0}\left(h y_{n}^{\prime}+\frac{1}{2} h^{2} y_{n}^{\prime \prime}+\frac{1}{6} h^{3} y_{n}^{(3)}-\frac{5 h^{4}\left(y_{n}^{(4)}\right)^{2}}{24\left(h y_{n}^{(5)}-5 y_{n}^{(4)}\right)}\right)=0
$$

the above indicates that the constructed schemes satisfy the consistency property.

\subsection{Stability}

To get the stability behaviour of the constructed scheme, the scheme is implemented on the standard test problem

$$
y^{\prime}=\lambda y, \quad \operatorname{Re}(\lambda)<0
$$

and the stability polynomial $R(z)=\frac{y_{n+1}}{y_{n}}$, where $z=\lambda h$ is obtained. The stability function of (5) is obtained as

$$
R(z)=\frac{y_{n+1}}{y_{n}}=\frac{-z^{4}-8 z^{3}-36 z^{2}-96 z-120}{24(z-5)}
$$

and the region of absolute stability is seen in Figure 1.

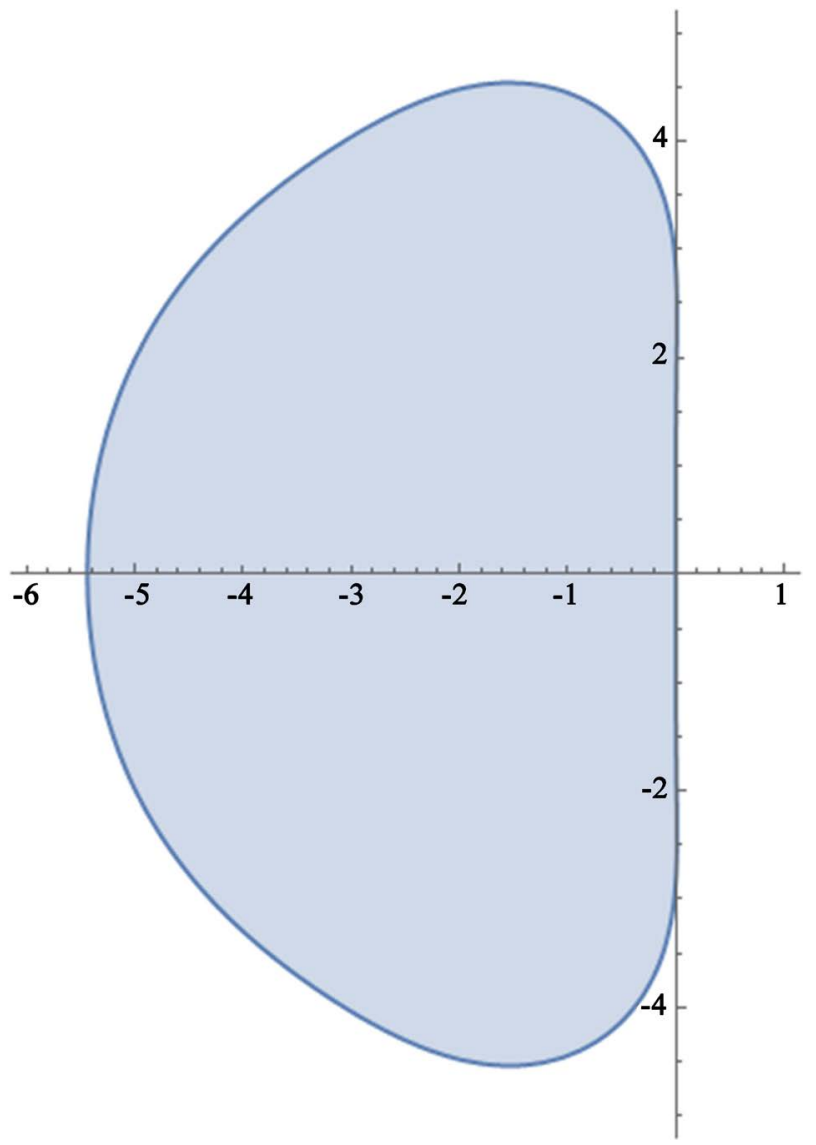

Figure 1. Region of absolute stability of (5). 


\section{Numerical Examples}

The first problem considered in this work is the nonlinear initial value problem

$$
y^{\prime}=1+y^{2} ; \quad y(0)=1
$$

whose theoretical solution is given as

$$
y(x)=\tan \left(x+\frac{\pi}{4}\right) .
$$

For this problem, the absolute errors of the results obtained by the method proposed in this work are first compared with those of Non-linear One-Step methods for initial value problems of [7] and the derivative-free methods proposed in [11] as shown in Figure 2. A comparison of the maximum absolute error obtained by the proposed methods against those produced by the methods of the authors in [4] [6] [7] [9] [13] is also presented in Figure 3.

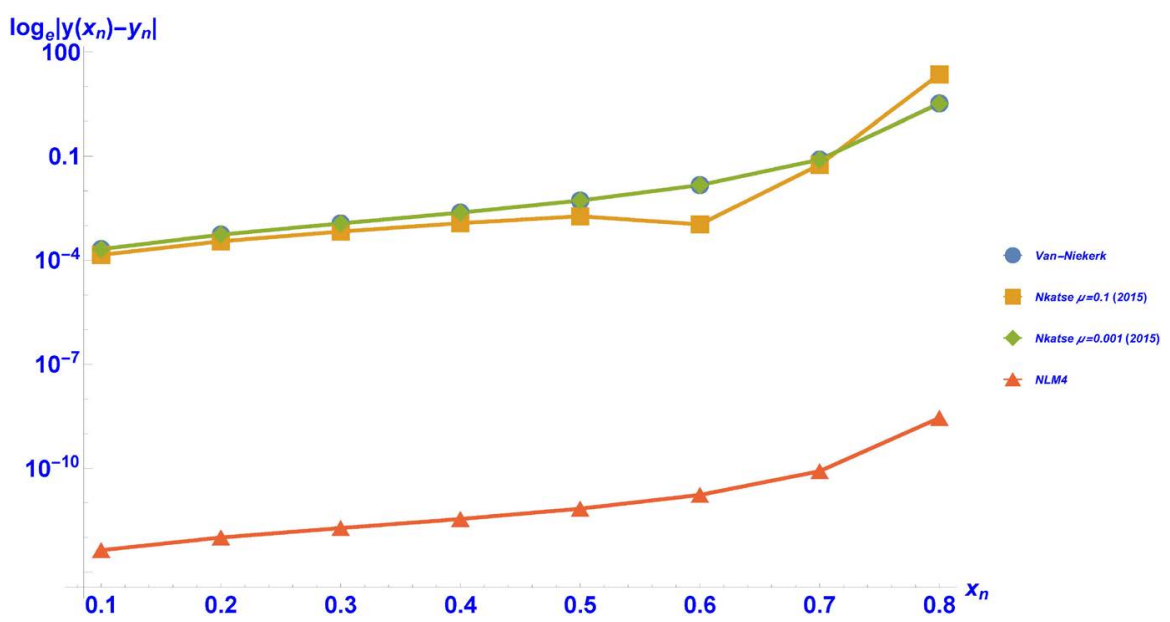

Figure 2. Logarithm of absolute errors for the solutions of Problem 1 with step-size $h=$ 0.01 .

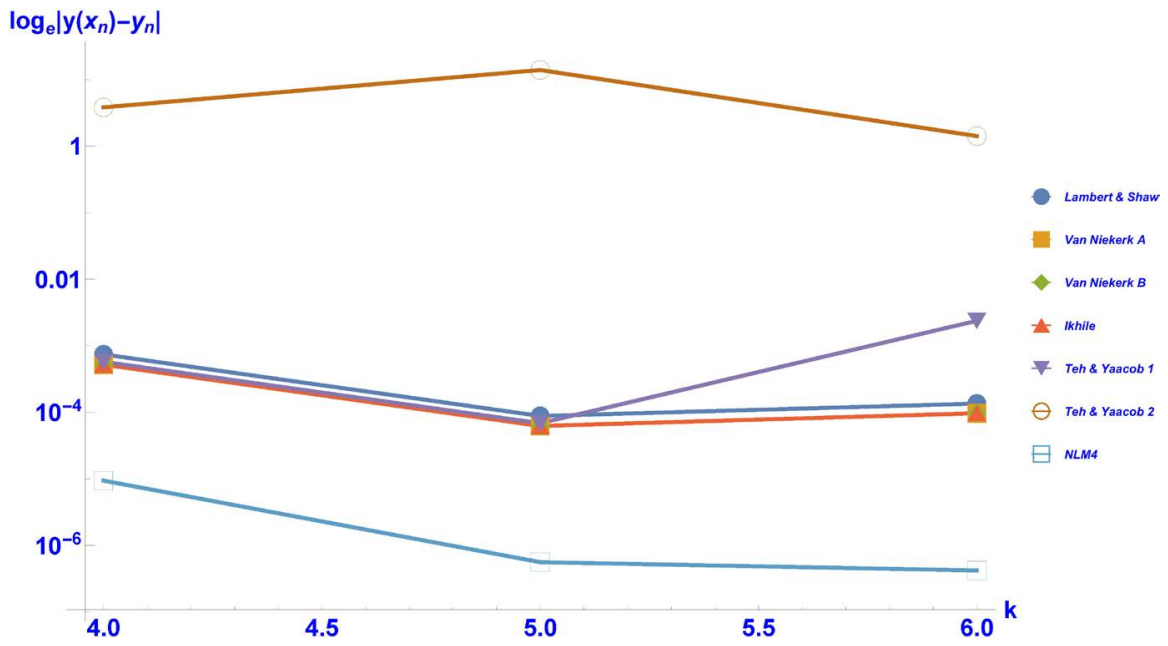

Figure 3. Log plot of maximum absolute errors for Problem 1 as a function of the step-size $h=\frac{0.8}{2^{k}}, k=4(1) 6$. 


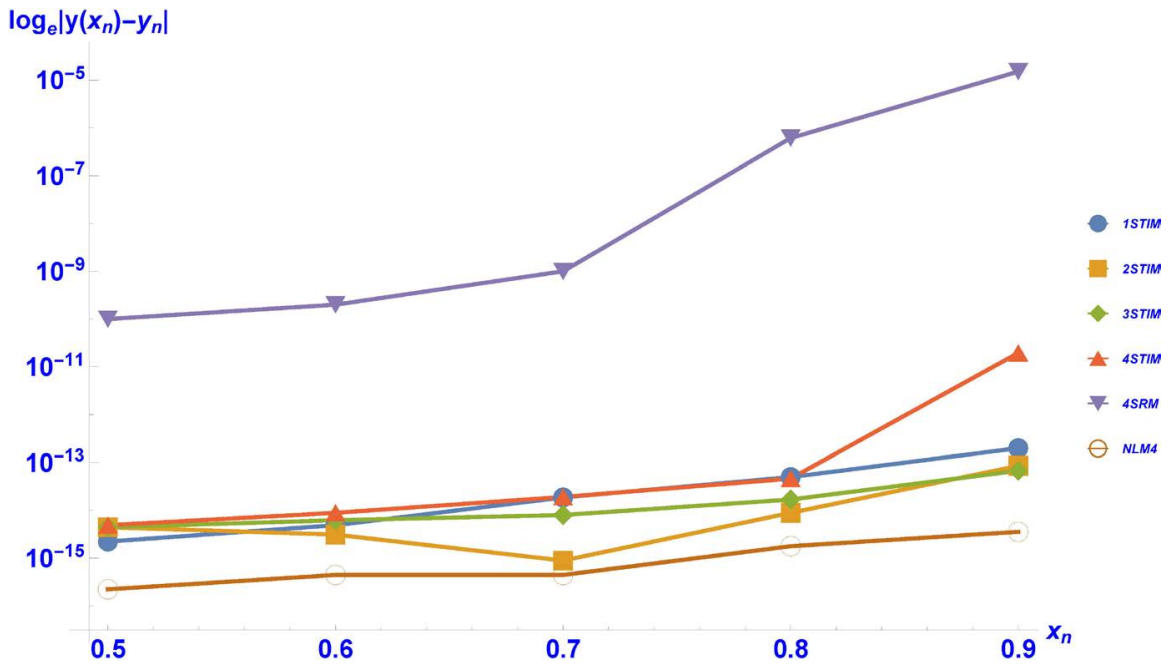

Figure 4. Logarithm of absolute errors for the solutions of (16) with step-size $h=0.01$.

\section{Problem 2}

The second test problem considered is given as

$$
y^{\prime}=y^{2} ; \quad y(0)=1 \text {. }
$$

The exact Solution is

$$
y(x)=\frac{1}{1-x}
$$

The logarithm of absolute errors for the solutions obtained is compared with other methods discussed in [12] as given in Figure 4.

\section{Conclusion}

The explicit single-step nonlinear method constructed in this work is consistent and absolutely stable. Its region of absolute stability is larger than those of the methods discussed in the literature. The method gave more accurate result on the standard test problems compared with other methods discussed. Hence, the method is suitable for solving problems whose solution possesses singularity.

\section{Conflicts of Interest}

The authors declare no conflicts of interest regarding the publication of this paper.

\section{References}

[1] Lambert, J.D. (1973) Computational Methods in Ordinary Differential Equations. Academic Press, Cambridge.

[2] Luke, Y.L., Fair, W. and Wimp, J. (1975) Predictor Corrector Formulas Based on Rational Interpolants. Computers \& Mathematics with Applications, 1, 3-12. https://doi.org/10.1016/0898-1221(75)90003-6

[3] Fatunla, S.O. (1991) Numerical Methods for IVPs in ODEs. Academic Press, New York. 
[4] Lambert, J.D. and Shaw, B. (1965) On the Numerical Solution of $y^{\prime}=f(x, y)$ by a Class of Formulae Based on Rational $y^{\prime}$ Approximation. Mathematics of Computation, 19, 456-462. https://doi.org/10.2307/2003678

[5] Fatunla, S.O. (1986) Numerical Treatment of Singular Initial Value Problems. An International Journal of Computers and Mathematics with Applications, 128, 1109-1115. https://doi.org/10.1016/0898-1221(86)90235-X

[6] Ikhile, M.N.O. (2001) Coefficients for Studying One-Step Rational Schemes for IVPs in ODEs. Computers and Mathematics with Applications, 41, 769-781. https://doi.org/10.1016/S0898-1221(00)00319-9

[7] van Niekerk, F.D. (1987) Non-Linear One-Step Methods for Initial Value Problems. Journal of Computational and Applied Mathematics, 13, 367-371. https://doi.org/10.1016/0898-1221(87)90004-6

[8] Niekiek, F.D.V. (1988) Rational One Step Methods for Initial Value Problems, Computers \& Mathematics with Applications, 16, 1035-1039.

https://doi.org/10.1016/0898-1221(88)90259-3

[9] Teh, Y.Y. and Yaacob, N. (2013) One-Step Exponential-Rational Methods for the Numerical Solution of First Order Initial Value Problems. Sains Malaysiana, 42, 456-462.

[10] Fatunla, S.O. (1982) Nonlinear Multistep Methods for Initial Value Problems. An International Journal of Computers and Mathematics with Applications, 8, 231-239. https://doi.org/10.1016/0898-1221(82)90046-3

[11] Nkatse, T. and Tshelametse, R. (2015) Analysis of Derivative Free Rational Scheme. MATEMATIKA, 31, 135-142.

[12] Tasneem, A., Asif, A.S. and Sania, Q. (2018) Development of a Nonlinear Hybrid Numerical Method. Advances in Differential Equations and Control Process, 19, 275-285. https://doi.org/10.17654/DE019030275

[13] Ying, T.Y., Omar, Z. and Mansor, K.H. (2014) Modified Exponential-Rational Methods for the Numerical Solution of First Order Initial Value Problems. Sains Malaysiana, 43, 1951-1959. https://doi.org/10.17576/jsm-2014-4312-18 